\title{
Shallow donor states near a semiconductor-insulator-metal interface
}

\author{
Y. L. Hao, ${ }^{1}$ A. P. Djotyan, ${ }^{2}$ A. A. Avetisyan,,${ }^{1,2}$ and F. M. Peeters ${ }^{1,3, *}$ \\ ${ }^{1}$ Department of Physics, University of Antwerpen, Groenenborgerlaan 171, B-2020 Antwerpen, Belgium \\ ${ }^{2}$ Department of Physics, Yerevan State University, A. Manoogian 1, Yerevan 0025, Armenia \\ ${ }^{3}$ Departamento de Física, Universidade Federal do Ceará, Caixa Postal 6030, Campus do Pici, Fortaleza 60455-900, CE, Brazil
}

(Received 7 April 2009; revised manuscript received 8 July 2009; published 30 July 2009)

\begin{abstract}
The lowest energy electronic states of a donor located near a semiconductor-insulator-metal interface are investigated within the effective mass approach. The effect of the finite thickness of the insulator between the semiconductor and the metallic gate on the energy levels is studied. The lowest energy states are obtained through a variational approach, which takes into account the influence of all image charges that arise due to the presence of the metallic and the dielectric interfaces. We compare our results with a numerical exact calculation using the finite element technique.
\end{abstract}

DOI: 10.1103/PhysRevB.80.035329

PACS number(s): 73.20.Hb

\section{INTRODUCTION}

It is well known that the electrical and optical properties of bulk and low dimensional semiconductors strongly depend on the presence of donor impurities. Recently there has been an enhanced interest to study shallow donor impurities near an interface (either with vacuum or at a metal-oxidesemiconductor interface) because of its importance in atomic scale electronics and nanoelectronics. This is a consequence of the growing tendency for miniaturization of electronic devices. ${ }^{1}$ Doped $\mathrm{Si}$ is a promising candidate for quantum computing due to its scalability, long spin coherence time, and the amazing progress in Si technology. ${ }^{2}$ The precise control of the dopant position is necessary for the experimental realization of such a working qubit. There are increasing efforts to reach the required accuracy in donor positioning using top-down techniques ${ }^{3-5}$ (single-ion implantation with $\mathrm{nm}$ accuracy), as well as using scanning tunnel microscopy (STM), i.e., positioning of P donors on a monohydride surface with $1 \mathrm{~nm}$ accuracy with subsequent $\mathrm{Si}$ overgrowth. ${ }^{6,7}$ For fundamental physics as well as for applications it is interesting to develop analytical methods for a better understanding of the physics of single dopants localized near an interface taking into account the complete electrostatics of the problem, i.e., the image charges.

For the case of a semiconductor/metal interface the energy spectrum was recently investigated in Ref. 8 using the finite element technique. In Ref. 9 the ground state energy of a donor localized in Si near an interface with a thick insulating layer (semiconductor/dielectric system) was studied using a variational approach. For intermediate and large values of the distance between the donor and the interface a reasonable agreement was found with the results for the ground state energy obtained in Ref. 10 where a variational method was applied with a trial wave function consisting of a summation over a basis set of wave functions.

Due to the increased miniaturization the oxide layer between the semiconductor and the metallic gate is strongly reduced in thickness. Therefore, it becomes important to include the screening effect of the metallic gate on the impurity states that are localized near the semiconductor/insulator interface. This motivated us to develop a variational approach for shallow donor states localized in a semiconductor at a semiconductor-insulator-metal interface. We introduce a term in the variational wave function that is responsible for the interaction of the electron with the images in the insulator and/or in the metallic gate which leads to a considerable lowering of the energy, especially when the impurity is located very near the interface. Our results for the ground and the first excited state energies of the impurity electron bound to the donor as obtained from our variational approach are in a good agreement with the "numerically exact" energies found using the numerical finite element approach for a semiconductor/metal and especially for a semiconductor/ dielectric system. For the latter case we compare the ground state energy also with previous theoretical work of MacMillen and Landman. ${ }^{10}$ We found that for the case of equal as well as for the case of different effective masses in parallel and perpendicular directions to the interface our method gives lower energy.

The proposed variational functions are also applied to the semiconductor/insulator/metal interface problem with a finite width dielectric layer. Now the image charge problem leads to an infinite series of images. ${ }^{11}$ We also use a numerical finite element approach to obtain the energy levels numerically exact in order to have an idea of the accuracy of our variational approach. Because of the good agreement with the exact numerical solution we may conclude that our variational functions take into account the influence of all image charges.

The present paper is organized as follows. In Sec. II we propose trial wave functions for an impurity electron near a semiconductor/metal interface and the discussion of the results is presented in the same section. In Sec. III impurity states at a semiconductor/dielectric interface are studied on the basis of the same trial wave functions, introduced in Sec. II, and the appropriateness of the wave functions as well as the corresponding results are discussed there. A semiconductor/insulator/metal interface problem with a finite width dielectric layer is investigated in Sec. IV and the results obtained on the basis of the variational and numerical "finite element" method are presented in the same section. The conclusion is presented in Sec. V. 


\section{IMPURITY NEAR A SEMICONDUCTOR/METAL INTERFACE}

In this section we consider a donor at a semiconductor/ insulator/metal interface where we assume that the oxide layer is very thin and its only effect is to prevent the electron to penetrate into the metal, i.e., it provides a very high potential barrier. The thin oxide layer does not contribute to any dielectric mismatch ${ }^{8}$ and only the metallic gate screens the Coulomb potential. The permittivity at the semiconductor side of the interface for typical semiconductors of interest is about 10 while it is infinite in the metal. The dielectric mismatch effects at the interface give rise to image charges that screen the potential between the electron and the impurity. The potential energy between the electron and the impurity near the semiconductor/metal interface at a position $\vec{r}_{d}$ $=(0, d)$ is given by ${ }^{8}$

$$
U_{\text {Coul }}(\vec{r})=\frac{e^{2}}{\varepsilon_{s}}\left[-\frac{1}{4 z}+\frac{1}{\sqrt{\rho^{2}+(z+d)^{2}}}-\frac{1}{\sqrt{\rho^{2}+(z-d)^{2}}}\right]
$$

with $\rho=\sqrt{x^{2}+y^{2}}$. The first term describes the attractive interaction between the electron and its image, the second term is due to the repulsive interaction between the electron and the donor image (as well as between the donor and the electron image), and the last term represents the normal impurityelectron interaction. The problem is cylindrically symmetric and we use cylindrical coordinates. In dimensionless units expressed in terms of the Bohr radius $a_{B}=\hbar^{2} \varepsilon_{S} / m_{\perp} e^{2}$ (where $\varepsilon_{s}$ is the permittivity at the semiconductor side and $m_{\perp}$ is the transverse effective mass) and twice the Rydberg energy $2 R^{*}=\hbar^{2} / m_{\perp} a_{B}^{2}$ the Hamiltonian of the system is

$$
H=-\frac{1}{2}\left[\frac{\partial^{2}}{\partial \rho^{2}}+\frac{1}{\rho} \frac{\partial}{\partial \rho}+\frac{m}{\rho^{2}}+\sigma \frac{\partial^{2}}{\partial z^{2}}\right]+U_{\text {Coul }}(\vec{r}),
$$

where $\sigma$ is the ratio between the transverse and longitudinal effective masses $m_{\|}$. We mostly use $\sigma=1$ in this paper (we will mention explicitly if we use a different value, i.e., $\sigma$ $=0.2079$, which is typical for $\mathrm{Si}$ ). In GaAs we have $a_{B}$ $=9.89 \mathrm{~nm}, 2 R^{*}=11.6 \mathrm{meV}$, and $\varepsilon_{s}=12.9$ while for Si with $m_{\perp}=0.19 m_{0}$ we have $a_{B}=3.31 \mathrm{~nm}, 2 R^{*}=36.5 \mathrm{meV}$, and $\varepsilon_{s}=11.9$.

For the ground state variational wave function we propose

$$
\psi_{0}=N z \exp \left(-\sqrt{\lambda^{2} \rho^{2}+\gamma^{2}(z-d)^{2}}\right) \exp (-\beta z),
$$

where the factor $z$ guarantees that the wave function satisfies the boundary condition $\psi(z=0)=0$ (Ref. 12); $\lambda, \gamma$, and $\beta$ are variational parameters. The exponential factor $f(\beta z)$ $=\exp (-\beta z)$ describes the effect of the image charges and $g[\lambda \rho, \gamma(z-d)]=\exp \left(-\sqrt{\lambda^{2} \rho^{2}+\gamma^{2}(z-d)^{2}}\right)$ takes into account the electron-donor interaction. The normalization constant is $N=\sqrt{1 / 2 \pi A}$ with

$$
A=\int_{0}^{\infty} \rho d \rho \int_{0}^{\infty} d z z^{2} \exp (-2 \delta) \exp (-2 \beta z),
$$

where $\delta=\sqrt{\lambda^{2} \rho^{2}+\gamma^{2}(z-d)^{2}}$.

The ground state energy as calculated with the trial function Eq. (3) is given by

$$
E_{1 s}=\left(T_{1}+T_{2}+C\right) / A \text {, }
$$

where

$$
\begin{aligned}
T_{1}= & \int_{0}^{\infty} \rho d \rho \int_{0}^{\infty} d z\left[\frac{2 \lambda^{2}+\gamma^{2}}{2 \delta}\right. \\
& \left.-(1+\delta) \frac{\lambda^{4} \rho^{2}+\gamma^{4}(z-d)^{2}}{2 \delta^{3}}\right] z^{2} \exp (-2 \beta z) \exp (-2 \delta), \\
T_{2}= & \int_{0}^{\infty} \rho d \rho \int_{0}^{\infty} d z\left[\frac{\gamma^{2}(z-d)}{\delta} \frac{\partial z \exp (-\beta z)}{\partial z}\right. \\
& \left.-\frac{1}{2} \frac{\partial^{2} z \exp (-\beta z)}{\partial z^{2}}\right] z \exp (-\beta z) \exp (-2 \delta),
\end{aligned}
$$

$$
C=\int_{0}^{\infty} \rho d \rho \int_{0}^{\infty} d z U_{\text {Coul }}(\rho, z) z^{2} \exp (-2 \beta z) \exp (-2 \delta) .
$$

If we use a hydrogenlike factor in Eq. (3) with only one parameter (i.e., we take $\gamma=\lambda$ ), then in the ground state energy, Eq. (5), we have to replace $T_{1}$ and $T_{2}$ by

$$
\begin{gathered}
T_{1}^{\prime}=\int_{0}^{\infty} \rho d \rho \int_{0}^{\infty} d z\left(\frac{\lambda^{2}}{\delta}-\frac{\lambda^{2}}{2}\right) z^{2} \exp (-2 \beta z) \exp (-2 \delta), \\
T_{2}^{\prime}=\int_{0}^{\infty} \rho d \rho \int_{0}^{\infty} d z\left[\frac{\lambda^{2}(z-d)}{\delta} \frac{\partial z \exp (-\beta z)}{\partial z}\right. \\
\left.-\frac{1}{2} \frac{\partial^{2} z \exp (-\beta z)}{\partial z^{2}}\right] z \exp (-\beta z) \exp (-2 \delta) .
\end{gathered}
$$

The numerical results for the two different variational wave functions in the case of equal transverse and longitudinal effective masses $(\sigma=1)$ are shown in Fig. 1 where we compare them with the results from an exact numerical diagonalization of the problem. For intermediate values of $d$ all three results are practically the same, e.g., for $d / a_{B}=3$ we found $E / 2 R^{*}=-0.420,-0.418,-0.416$ for, respectively, the exact, three-parameter and two-parameter trial function results. For small values of $d$ the differences are larger, e.g., for $d / a_{B}$ $=1$ we found $E / 2 R^{*}=-0.152,-0.142,-0.138$ for, respectively, the exact result, three-parameter and two-parameter trial functions. In Fig. 1 we also present the results when we remove the image charges and include only the direct Coulomb interaction between the electron and the donor and take into account the boundary condition at the surface. Notice that: (i) the binding energy is larger (typically $0.2 R^{*}$ for $d$ $<3 a_{B}$ ) implying that the metal screens the electron-donor Coulomb interaction and (ii) the $d \rightarrow \infty$ limit is reached from below while when including the image charges this limit is reached from above.

The profile of the impurity electron potential along the $z$ axis (with $\rho=0$ ) for the semiconductor/insulator/metal case with infinitely thin dielectric layer shown in Fig. 1 of Ref. 8 exhibits a double well structure and when the impurity moves toward the interface, the potential wells start to interact strongly and eventually combines into a single well. In this region of close distances of the donor from the interface 


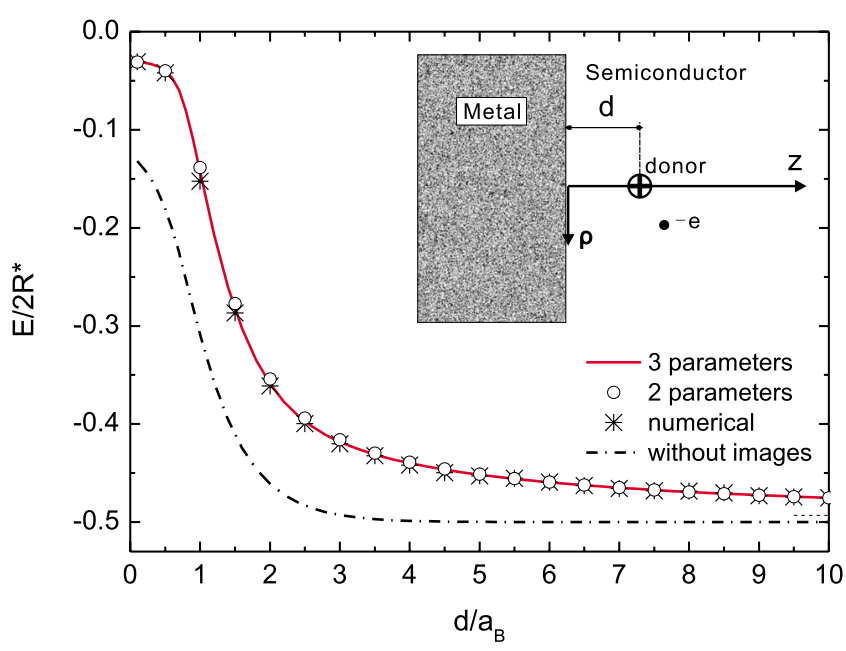

FIG. 1. (Color online) The dependence of the ground state energy (in units of $2 R^{*}$ ) vs the donor position $d / a_{B}$ with respect to the metal interface (for the semiconductor/metal interface problem). We compare the variational results with two parameters (circles), with the three variational parameter trial function (solid line) and "exact solution" (stars) found in Ref. 8. The thin dashed line is the result for $d=\infty$. For the comparison the ground state energy for the system in the absence of the image effects is also presented (dot-dashed curve). Inset: the configuration of the semiconductor/metal system.

the term $f(\beta z)=\exp (-\beta z)$ in the trial wave function is very important and leads for small distance $d \sim a_{B}$ to an energy lowering of more than $15 \%$; if we take $\beta=0$ in Eq. (3) (with $\gamma \neq \lambda$ ) the ground state energy becomes $E / 2 R^{*}=-0.119$. We compared our results with the numerical "exact" solution and obtained a good agreement: the relative error is $0.6 \%$ when we use the trial function with three variational parameters, i.e., with $\gamma \neq \lambda$ in the hydrogenlike function $g[\lambda \rho, \gamma(z-d)]$ in Eq. (3) for the impurity distance $d=2 a_{B}$ from the interface and becomes $1.9 \%$ for the two-parameter case, i.e., when $\gamma$ $=\lambda$. The relative error tends to zero when $d>3 a_{B}$ for both these cases. For smaller distances, when $d=a_{B}$ the relative error is about $6 \%(10 \%)$ when we use the trial function with three variational parameters (with two variational parameters). We see that for the case when the hydrogenlike factor in the trial function contains only one variational parameter, i.e., $\gamma=\lambda$ the relative error is about $10 \%$ for $d=a_{B}$. In this region the electron charge distribution is strongly nonspherical and one needs to introduce different variational parameters in the $\rho$ and $z$ directions in the hydrogenlike factor.

It is interesting to study this problem when the donor is very close to the metal interface with $d \sim 0$. In this case the potential energy Eq. (1) leads to $U_{\text {Coul }} \approx-1 / 4 z$ and becomes exactly equal to $U_{\text {Coul }}=-1 / 4 z$ when $d=0$ since in this case the effect of the donor on the electron is compensated by the donor image. Using Eq. (5) we found for $d \rightarrow 0$ that the variational parameters $\lambda, \gamma \rightarrow 0$ and $\beta \simeq 0.25$. For $d=0$ the electron is no longer bound to the donor but only to the interface and we have a strictly one-dimensional problem. In this region we can use for the trial function $\psi_{0}$ $=N z \exp (-\beta z)$, which gives for the ground state energy a simple expression

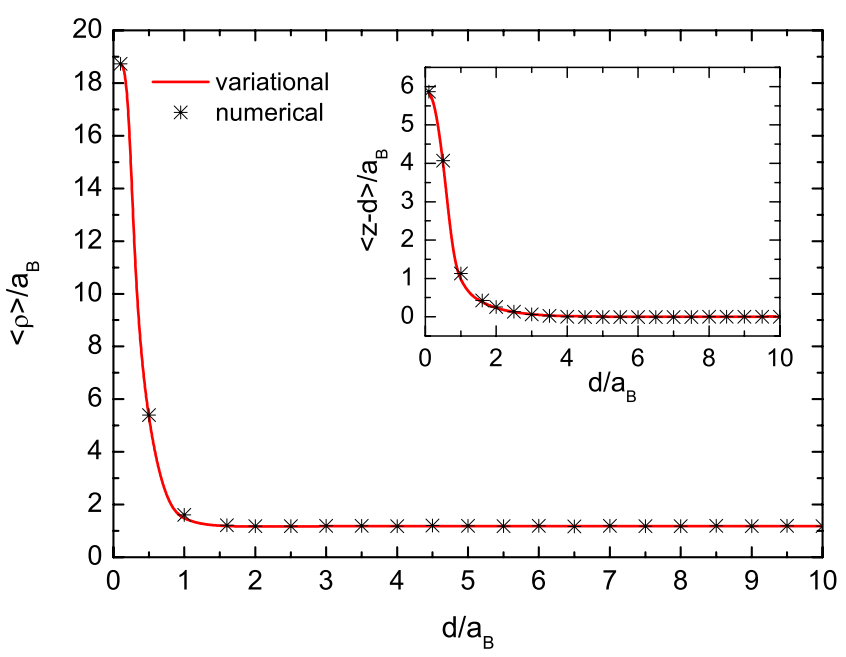

FIG. 2. (Color online) The average value of the electron position in $\rho$ direction in the $1 s$ state vs the donor position in units of $a_{B}$ for the semiconductor/metal interface. Inset: the dependence of the average distance between the electron and the donor along the $z$ direction.

$$
E_{0}=-\beta / 4+\beta^{2} / 2,
$$

which has the energy minimum $E_{0} / 2 R^{*}=-0.0312$ at $\beta$ $=0.25$.

From Eq. (5) we found numerically for $d \rightarrow 0$ a plateau in the value of the ground state energy with $E_{0} / 2 R^{*}=-0.033$ for $d=0.3 a_{B}$ and the relative error with the exact result is about $1 \%$ for these distances. The difference with the result from Eq. (7) for $d=0$ is $5 \%$ up to a distance of $d=0.3 a_{B}$.

In Fig. 2 we show the expectation value of the electron position in units of $a_{B}$ in the $\rho$ plane $\int \rho \psi_{0}^{2} d \mathbf{r}$ and as an inset of the figure the expectation value along the $z$ axis $\int(z-d) \psi_{0}^{2} d \mathbf{r}$. The ground state energy is very small for $d$ $\rightarrow 0$ since $\langle z\rangle$ is increased about six times in comparison with the bulk case and due to this the attractive Coulomb interaction is effectively reduced. Figure 2 also shows that for $d$ $\rightarrow 0$ the electron is spread out in $\langle\rho\rangle$ plane; when $d=0$ the probability to find the electron in different $\rho$ points is a constant and the coordinates $\rho$ and $z$ are decoupled. Notice that we have $\langle z\rangle=d$ for $d>4 a_{B}$ and $\langle\rho\rangle$ reaches its bulk value for $d>2 a_{B}$ while the energy is still influenced by the presence of the interface for these $d$ values. As it is well known, the ground state energy of the one-dimensional hydrogenlike atom does not exist, i.e., it is infinitely deep. ${ }^{13}$ But in our case the ground state energy of the impurity electron [Eq. (7)] has a finite value for $d=0$ due to the presence of an impenetrable interface.

Next we construct the trial wave function of the $2 s$ excited state, with the quantum numbers $(n, l, m)=(2,0,0)$ in the bulk case, where we took as variational wave function

$$
\psi_{2 s}=N z\left(1-\sqrt{\lambda^{2} \rho^{2}+\gamma^{2}(z-d)^{2}}-\alpha_{2 S} z\right) g[\lambda \rho, \gamma(z-d)] f(\beta z)
$$

and the parameter $\alpha_{2 S}$ is defined from the orthogonalization condition $^{14} \int \psi_{0}^{*} \psi_{2 s} d \mathbf{r}=0$. 


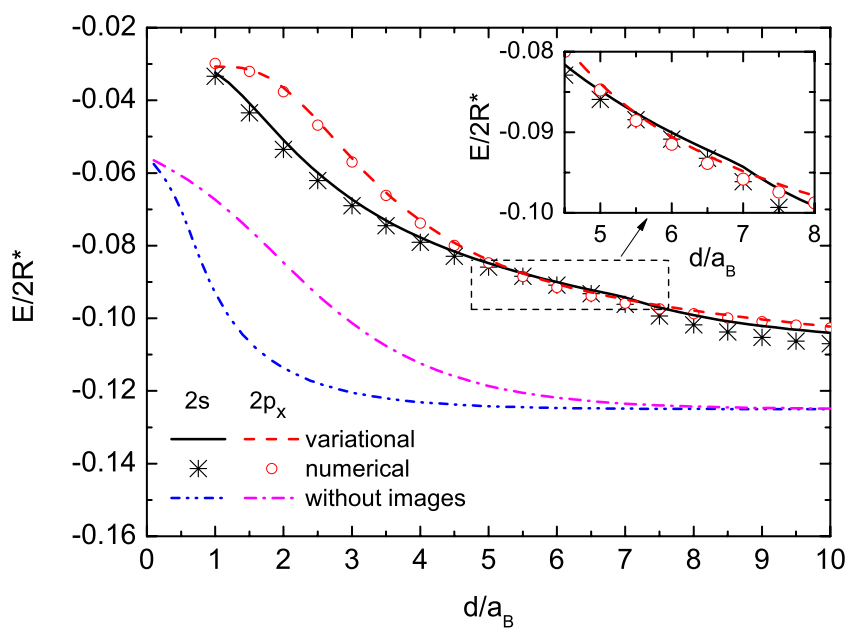

FIG. 3. (Color online) The dependence of the excited state energies $2 s$ (solid curve) and $2 p_{x}$ (dashed curve) calculated variationally (with three parameters), as well as the numerical exact result indicated by stars for $2 s$ and by circles for $2 p_{x}$ state (in units of $2 R^{*}$ ) vs the donor position $d / a_{B}$ from the metal gate (for the system with an infinitely thin insulator layer). The excited state energies $2 s$ (dotdot-dashed curve) and $2 p_{x}$ (dot-dashed curve) for the system in the absence of the image effects.

For the $(2,1, \pm 1)$ states we choose the following variational functions: ${ }^{14}$

$$
\begin{aligned}
& \psi_{2 p_{x}}=N z g[\lambda \rho, \gamma(z-d)] \rho \cos \varphi f(z), \\
& \psi_{2 p_{y}}=N z g[\lambda \rho, \gamma(z-d)] \rho \sin \varphi f(z) .
\end{aligned}
$$

Figure 3 shows the dependence of the energy of the excited states $2 s$ (solid curve) and $2 p_{x}$ (dashed curve) (in units of $2 R^{*}$ ) as a function of $d$ (in units of $a_{B}$ ), calculated with the three-parameter trial function Eq. (8). As a comparison, in the same figure we present the energy dependence for the $2 s$ state obtained in Ref. 8 indicated by stars as well as for the $2 p_{x}$ state which are given by circles. The excited state energies in the absence of the image effects are also presented in Fig. 3.

The comparison of the energy for the excited states $2 s$ and $2 p_{x}$ calculated by the variational approach with the results obtained with the finite element method gives a relative error that is less than $5 \%$, even for the case when the donor is close to the interface with $d=a_{B}$. Notice that the presence of the interface lifts the degeneracy of the $2 s$ and $2 p_{x}$ states and we have $E_{2 p_{x}}>E_{2 s}$ except around $d \sim 6 a_{B}$ where we find a region with $E_{2 p_{x}}$ slightly lower energy than $E_{2 s}$. This crossing behavior of the two energy levels was studied in detail in Ref. 8. Notice that the crossing points depend strongly on the accuracy of the used variational wave functions. In Ref. 8 the electron probability density was studied for the excited states. There it was found that near the first crossing point at $d \sim 7 a_{B}$ for the $2 s$ state the probability density is larger in the region $z<d$. The reason is that even for such a large $d$ the electron has a finite probability to be near the interface since in an excited state the electron is less strong bound to the donor and is still appreciably attracted by the image resulting in $\langle z\rangle<d$ and a $\langle\rho\rangle$ which is larger than its corresponding bulk value. For smaller $d$ values the effect of the interface as well as the repulsive interaction between the electron and the donor image increases; at $d \sim 6.5 a_{B}$ we find also on the basis of our variational method that $\langle z\rangle \sim d$ and $\langle\rho\rangle$ is almost decreased to its bulk value. With a further decrease in $d$ we find that $\langle z\rangle$ and $\langle\rho\rangle$ are monotonically increasing. When we neglect the image charges: (i) the binding energies of the $2 s$ and $2 p$ states are two times larger for $d<3 a_{B}$; (ii) for $d$ $\sim 10 a_{B}$ the binding energy is still about $20 \%$ larger as compared to the fully screened problem. Notice that both results converge very slowly to each other with increasing $d$; and (iii) there is no crossing between the $2 s$ and $2 p$ binding energies.

\section{IMPURITY NEAR A SEMICONDUCTOR/INSULATOR INTERFACE}

In the limiting case of a very thick dielectric layer (what implies the absence of a metallic gate) only the oxide layer screens the Coulomb potential and the potential energy between the electron and the donor located at a distance $d$ from the semiconductor/dielectric interface takes the form (when using dimensionless units of $a_{B}$ and $R^{*}$ ),

$$
U_{\text {Coul }}(\vec{r})=\frac{Q}{4 z}-\frac{Q}{\sqrt{\rho^{2}+(z+d)^{2}}}-\frac{1}{\sqrt{\rho^{2}+(z-d)^{2}}},
$$

where $Q=\left(\varepsilon_{s}-\varepsilon_{o x}\right) /\left(\varepsilon_{s}+\varepsilon_{o x}\right)>0$ with $\varepsilon_{s}$ permittivity at the semiconductor side of the interface and $\varepsilon_{o x}$ for the dielectric. Now, the first term describes the repulsive interaction between the electron and its image, the second term is due to the attractive interaction between the electron and the donor image (as well as between the donor and the electron image), and the last term represents the direct impurity-electron Coulomb interaction. For this problem we choose the same trial wave function Eq. (3) which is motivated as follows. The Coulomb correlation between two negatively charged particles (the electron and its image) for the system with the potential energy Eq. (9) can be described by a factor $(1+\delta z)$. Similar functions for the electron-electron repulsive interaction were used previously for different systems (quantum wells ${ }^{15}$ and $\operatorname{dots}^{16}$ ). If we assume that the electron and the donor image interact only along the $z$ direction, then this and the interaction between the electron and its image can be taken care of using the exponential factor $f(z)=\exp (-\beta z)$; the validity of this approximation is confirmed by a comparison with the exact solution.

For the case of a semiconductor/dielectric interface we compared our results with the theoretical results of MacMillen and Landman ${ }^{10}$ presented in Table I, which was obtained for a "perfect imaging plane," i.e., with $Q=1$ and for the case of equal effective masses in parallel and perpendicular directions to the interface $(\sigma=1)$. We found that our method gives lower energy even in the case when the donor is localized at small distance from the interface. Here, the factor $f(z)$ leads to an energy lowering of about $5 \%$ for the donor distance $d=a_{B}$.

Now, we consider the case of nonequal effective masses in parallel and perpendicular directions to the interface with 
TABLE I. The values of the variational parameters $\lambda$ and $\beta$ and the ground state energy $E_{0}$ of the impurity electron near the semiconductor/insulator interface for different values of $d / a_{B}$ calculated on the basis of the variational wave function Eq. (3) with $\lambda=\gamma$, and the result $E_{0}^{\prime}$ found in Ref. 10 for $\sigma=1$ (with $Q=1$ ) as well as the relative error between these two results defined as $\left(E_{0}-E_{0}^{\prime}\right) / E_{0}$ are given.

\begin{tabular}{cccccc}
\hline \hline$d / a_{B}$ & $\lambda$ & $\beta$ & $E_{0} / R^{*}$ present & $E_{0}^{\prime} / R^{*}$ Ref. 10 & Relative error in $\%$ \\
\hline 0.4 & 0.797 & 0.011 & -0.716 & -0.651 & 9.9 \\
1.0 & 0.971 & 0.280 & -0.927 & -0.895 & 3.6 \\
1.6 & 1.004 & 0.370 & -1.077 & -1.052 & 2.4 \\
2.0 & 0.992 & 0.366 & -1.116 & -1.093 & 2.1 \\
3.0 & 0.969 & 0.306 & -1.128 & -1.109 & 1.7 \\
4.0 & 0.968 & 0.246 & -1.111 & -1.094 & 1.6 \\
6.0 & 0.979 & 0.168 & -1.080 & -1.068 & 1.1 \\
\hline \hline
\end{tabular}

$\sigma=0.2079$ as it is for $\mathrm{Si}$. The ground state energy of the system with $\sigma=0.2079$ when the donor is localized at the distances $d<3 a_{B}$ from the semiconductor-dielectric interface found in Ref. 9, using a variational approach, has much higher value than those found in Ref. 10. Only for large values of $d$ both results agree. The results found on the basis of our three-parameter variational wave function and Eq. (2) with $\sigma=0.2079$, as well as the results of Refs. 9 and 10 are compared in Table II. For the case of the different masses $f(z)$ leads to an energy lowering of about $6 \%$ when $d=a_{B}$.

In the rest of our paper we restrict ourselves to the case $\sigma=1$. In Fig. 4 we present the dependence of the ground state energy (in units of $2 R^{*}$ ) as a function of the distance $d$ of the donor from the semiconductor/dielectric interface (in units of $a_{B}$ ) calculated with the three variational parameter trial function Eq. (3) (solid curve). In the same figure the ground state energy dependence calculated with the trial function Eq. (3), in which we took $\gamma=\lambda$, is indicated by circles as well as by stars for the exact numerical results. We used material constants for $\mathrm{Si}$ with $\varepsilon_{s}=11.9$ and for the oxide side $\varepsilon_{o x}=3.4$, which is for $\mathrm{SiO}_{2}$. The ground state energy in the absence of the image effects is also presented in Fig. 4 (dot-dashed curve). Notice that now the image charges lead to an increase in the binding energy which for small $d$ amounts to almost a factor of 2 increase. Thus in this case we have an effective enhancement (i.e., antiscreening) of the electron-donor interaction.

To estimate the large $d$ contribution of the image terms [first and second terms in Eq. (9)] in the energy of the system we substitute into Eq. (9) $z \approx d \gg \rho$ which leads to $\Delta E / 2 R^{*}$
$\sim-Q / 4 d$. For $d \approx 10 a_{B}$ this gives an energy difference of $\Delta E / 2 R^{*} \sim-0.014$ which is very close to the distance between solid and dot-dashed curves in Fig. 4. For the semiconductor/metal interface case $(Q=-1)$ at $d=10 a_{B}$ $\Delta E / 2 R^{*} \sim 1 / 4 d=0.025$ and is in a very good agreement with the corresponding numerical result.

For large distances $d \rightarrow \infty$ the electron interacts only with the donor and we found that the ground as well as excited energies tend to the corresponding bulk limits. For intermediate distances $d$ the electron starts to feel also the donor image and a minimum at $d=3.25 a_{B}$ with $E_{0} / 2 R^{*}=-0.533$ appears in Fig. 4 (with $\lambda=0.99, \gamma=0.85$, and $\beta=0.32$ ). Notice that such a local minimum is absent when we do not include the image charges in our calculation.

In Fig. 5 we present the expectation value of the electron position in the ground state along the $z$ axis $\int(z-d) \psi_{0}^{2} d \mathbf{r}$ and in the $\rho$ plane $\int \rho \psi_{0}^{2} d \mathbf{r}$. Notice that the electron is localized on the impurity, i.e., $\langle z\rangle=d$ for $d>6 a_{B} ;\langle\rho\rangle$ has a local minimum for $d \sim 1.5 a_{B}$ and $\langle\rho\rangle$ reaches its bulk value from below for $d>6 a_{B}$. Figure 5 shows that $\langle\rho\rangle$ is decreased at $d \sim 3 a_{B}$ (where the ground state energy has a local minimum) and $\langle z\rangle$ is increased in comparison with their bulk values. Due to the squeezing along the $z$ axis at $d \sim 3 a_{B}$ the electron image is partially screened by the donor image while the electron is bound to the donor and its image. As a result the system gains some additional binding. Until $d=1.5 a_{B}$ the electron wave function in the lateral direction is further squeezed which reduces the repulsive interaction between the electron and its image but in this case the energy increases since $\langle z\rangle$ increases rapidly with decreasing $d$ (mainly as an effect of

TABLE II. The ground state energy $E_{0}$ of the impurity electron near the semiconductor/insulator interface for different values of $d / a_{B}$ calculated for the case $\sigma=0.2079(Q=1)$ on the basis of the three-parameter variational wave function Eq. (3) and the energy $E_{0}^{\prime}$ found in Ref. 10 as well as the energy $E_{0}^{\prime \prime}$ found in Ref. 9.

\begin{tabular}{ccccccc}
\hline \hline$d / a_{B}$ & $\lambda$ & $\gamma$ & $\beta$ & $E_{0} / R^{*}$ present & $E_{0}^{\prime} / R^{*}$ Ref. 10 & $E_{0}^{\prime \prime} / R^{*}$ Ref. 9 \\
\hline 0.4 & 2.100 & 4.847 & 0.651 & -1.686 & -1.593 & \\
1.0 & 1.916 & 4.518 & 0.791 & -1.886 & -1.828 & -1.376 \\
2.0 & 1.799 & 5.045 & 0.491 & -1.793 & -1.757 & -1.527 \\
3.0 & 1.785 & 5.211 & 0.335 & -1.726 & -1.701 & -1.552 \\
4.0 & 1.783 & 5.289 & 0.251 & -1.688 & -1.670 & -1.555 \\
\hline \hline
\end{tabular}




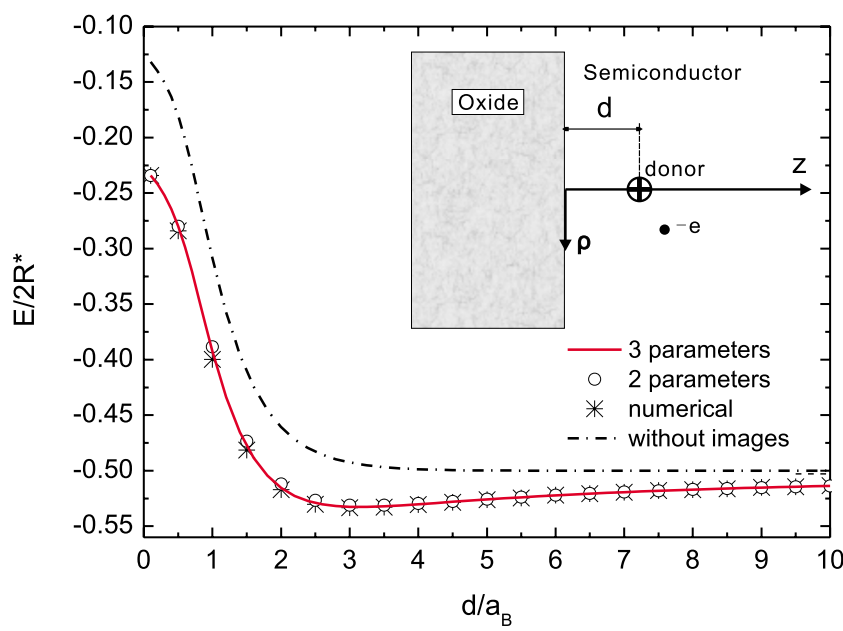

FIG. 4. (Color online) The dependence of the ground state energy (in units of $2 R^{*}$ ) as a function of $d / a_{B}$ with respect to the semiconductor/dielectric interface using the trial function with three variational parameters (solid curve). As a comparison we indicate by circles the results obtained with the two parameters trial function as well as by stars the exact results. The result for $d \rightarrow \infty$ is indicated by the dashed horizontal line. We present also the ground state energy in the absence of the image effects (dot-dashed curve). Inset: the configuration of the semiconductor/dielectric system.

the interface and of the repulsive Coulomb interaction). Figure 5 shows also that $\langle\rho\rangle$ is also increasing with further decrease in $d$.

Our variational approach gives very good agreement with the exact numerical results based on the finite element method. For $d \geq 2 a_{B}$ the two-parameter and three-parameter variational functions give practically the same results with the exact result. The relative error is only about $1 \%$ for $d$ $=a_{B}$, when we use Eq. (3) with three variational parameters which leads to the energy $E_{0} / 2 R^{*}=-0.392$ (with $\lambda=0.92, \gamma$ $=0.67$, and $\beta=0.38)$.

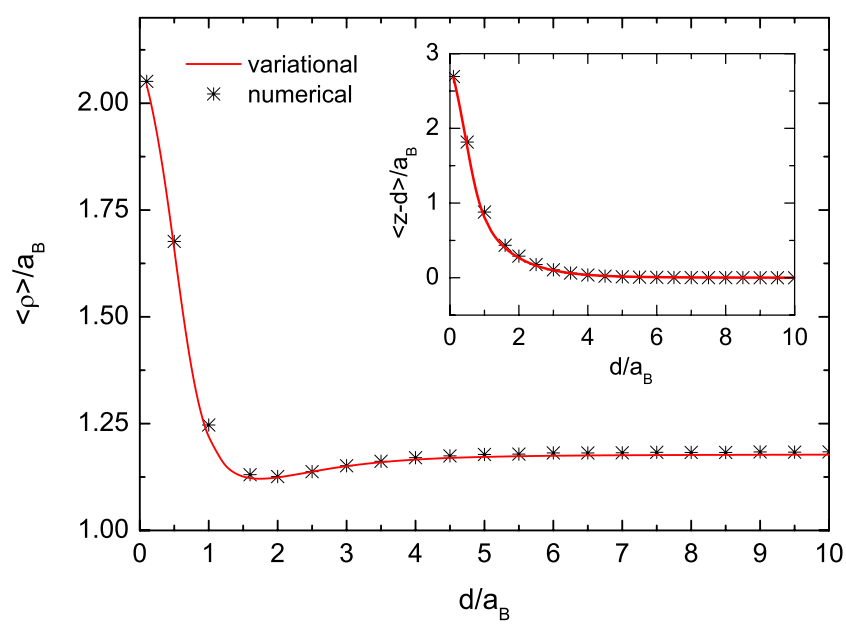

FIG. 5. (Color online) The average value of the electron position in the lateral direction for the $1 s$ state vs the donor position in units of $a_{B}$ for the case of semiconductor/dielectric interface. Inset: the dependence of the average distance between the electron and the donor along the $z$ direction.

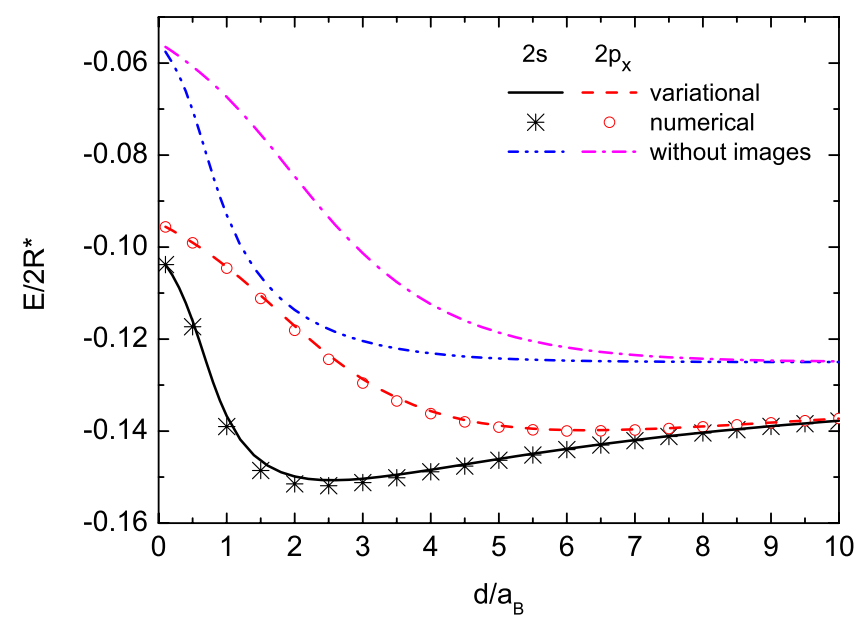

FIG. 6. (Color online) The dependence of the excited state energies $2 s$ (solid curve) and $2 p_{x}$ (dashed curve) calculated variationally and numerical exact indicated by stars for $2 s$ and by circles for $2 p_{x}$ (in units of $2 R^{*}$ ) as a function $d / a_{B}$ for a semiconductor/ dielectric system. The excited state energies for $2 s$ (dot-dot-dashed curve) and $2 p_{x}$ (dot-dashed curve) for the system in the absence of the image effects.

Since the interaction between the electron and its image is repulsive for the semiconductor/dielectric system, the electron wave function is not spread out at the interface when the donor is close to the interface, and the trial function Eq. (3) even with one variational parameter in the hydrogeniclike function $\exp \left(-\lambda \sqrt{r^{2}+(z-d)^{2}}\right)$ leads to the ground state energy with a relative error of about $2 \%$ when $d=a_{B}$.

We are observing that $\beta$ changes its sign at a small values of $d$, namely, at $d \approx 0.1 a_{B}$. This fact is connected with the following: when the donor and its image are very close to each other the electron feels them as one point charge located at $z=0$, and now the factor $f(z)=\exp (-\beta z)$ describes mainly the repulsive interaction between the electron and its image [it can be presented as $f(z)=(1+|\beta| z)$ (Refs. 15 and 16)]. Consequently, the interactions of the electron with the donor and its image are described by the hydrogenic factor $g[\lambda \rho, \gamma(z-d)]$ in Eq. (3) with close values of $\lambda$ and $\gamma(\lambda$ $=0.55, \gamma=0.59)$.

Figure 6 shows the dependence of the energy of the excited states $2 s$ (solid curve) and $2 p_{x}$ (dashed curve) (in units of $2 R^{*}$ ) as a function of $d$ (in units of $a_{B}$ ) for a semiconductor/dielectric interface calculated with the threeparameter trial function Eq. (8). As a comparison we also show the numerical exact result for the $2 s\left(2 p_{x}\right)$ state indicated by stars (circles) as well as the energies in the absence of the image effects. Notice that now $E_{2 p_{x}}>E_{2 s}$ for all values of $d$ and there is no crossing of these two energy levels as in previous case. This is due to the fact that in the present case of a semiconductor/dielectric interface, the interaction between the electron and its image is repulsive (see Fig. 7) and consequently there is no potential well as in the semiconductor/metal case. The expectation values $\langle z\rangle$ and $\langle\rho\rangle$ as a function of $d$ have the same behavior as for the ground state, with the only difference that now for the $2 s$ state the minimum in the energy and in $\langle\rho\rangle$ occur almost at the same position, i.e., $d \sim 2.5 a_{B}$. Notice that: (i) also for the excited 


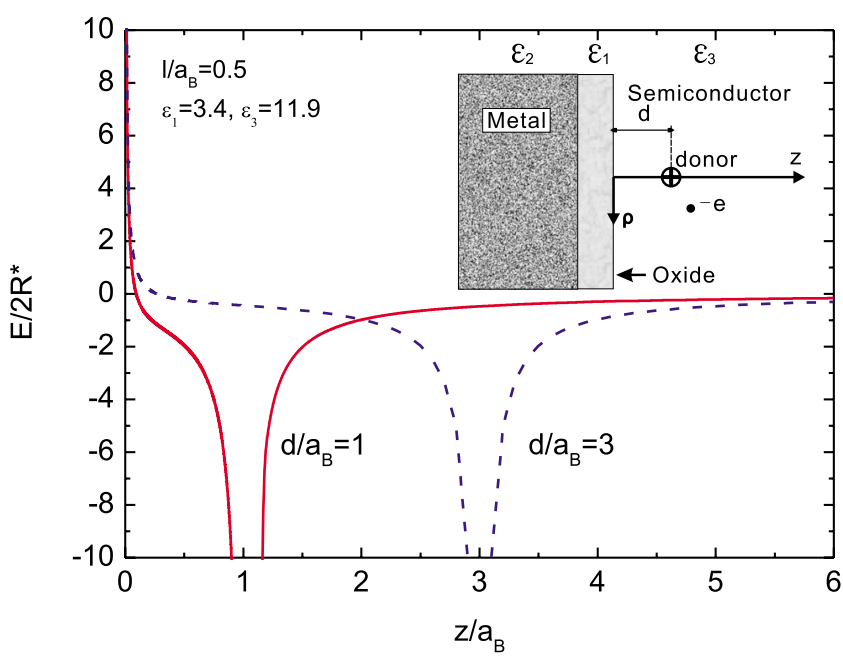

FIG. 7. (Color online) The potential energy (in units of $2 R^{*}$ ) of the electron along the $z$ axis (with $\rho=0$ ) for a semiconductor/ dielectric/metal interface with the oxide layer thickness $l / a_{B}=0.5$; solid line (dashed line) corresponds to the case when the impurity is located at the distance $d / a_{B}=1\left(d / a_{B}=3\right)$. Inset: the configuration of the semiconductor/insulator/metal system.

states there is almost a factor of 2 increase in the binding energy for small $d$ values when the image charge effect is included; (ii) the $2 s$ state without image charge effect does not exhibit a local minimum; and (iii) the result with image charge effect for large $d$ converges very slowly to the result without image charges.

\section{IMPURITY NEAR A SEMICONDUCTOR/INSULATOR/ METAL INTERFACE}

In this section we investigate the energy states of a shallow impurity in a semiconductor near an interface with a dielectric layer of finite thickness which is subsequently covered by a metallic layer. First we calculate the potential for the impurity in the case of three-layer problem using the image method. The system to be investigated is shown in the inset of Fig. 7, the impurity is located in the semiconductor at a distance $d$ from the interface.

Using the image method we find an infinite number of image charges for the electron ${ }^{11}$

$$
\begin{gathered}
e_{1}=e\left(\varepsilon_{3}-\varepsilon_{1}\right) /\left(\varepsilon_{3}+\varepsilon_{1}\right), \\
e_{-2 n-1}=e\left(\frac{\varepsilon_{1}-\varepsilon_{2}}{\varepsilon_{1}+\varepsilon_{2}} \frac{\varepsilon_{1}-\varepsilon_{3}}{\varepsilon_{1}+\varepsilon_{3}}\right)^{n} \frac{4 \varepsilon_{1} \varepsilon_{3}}{\left(\varepsilon_{1}+\varepsilon_{3}\right)^{2}} \frac{\varepsilon_{1}-\varepsilon_{2}}{\varepsilon_{1}+\varepsilon_{2}} \quad(n=0,1, \ldots),
\end{gathered}
$$

which are located at

$$
\begin{gathered}
z_{1}=-z_{e}, \\
z_{-2 n-1}=-(2 n+2) l-z_{e},
\end{gathered}
$$

where $l$ is the thickness of the oxide layer, and $e_{1}$ and $e_{-1}$ are the nearest image charges of the electron. Now, the potential energy (see Fig. 7) becomes

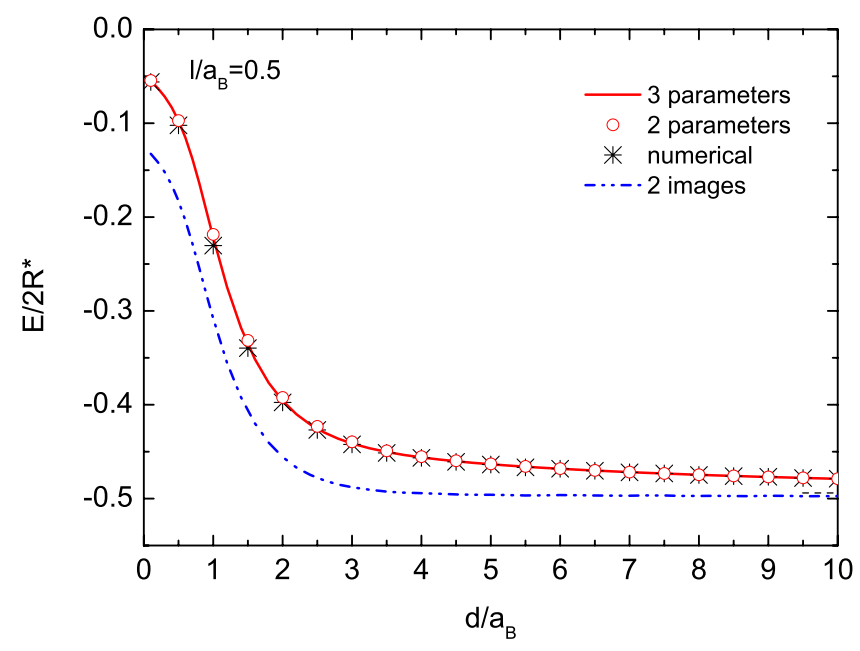

FIG. 8. (Color online) The dependence of the ground state energy (in units of $2 R^{*}$ ) as a function of $d / a_{B}$ with respect to the semiconductor/dielectric/metal interface, with the oxide layer thickness $l / a_{B}=0.5$, using the finite element techniques (stars) also from the trial function with three variational parameters (solid curve) and two parameters (circles). The thin dashed line is the result for $d$ $=\infty$. The ground state energy $\left(l / a_{B}=0.5\right)$ for the case when for each of the donor and the electron we take only two images (dot-dotdashed curve).

$$
\begin{aligned}
U_{\text {Coul }}^{3 \text { layer }}(\vec{r})= & \frac{1}{\varepsilon_{1}}\left[\sum_{n=0}^{\infty} \frac{e e_{-2 n+1}}{2\left|z_{e}-z_{-2 n+1}\right|}-\sum_{n=0}^{\infty} \frac{e e_{-2 n+1}}{\sqrt{\left(d-z_{-2 n+1}\right)^{2}+\rho_{e}^{2}}}\right. \\
& \left.-\frac{e^{2}}{\sqrt{\left(z_{e}-d\right)^{2}+\rho_{e}^{2}}}\right] .
\end{aligned}
$$

The first sum describes the interaction between the electron and its images, the second sum is due to the interaction between the electron and the impurity images (as well as between the impurity and the electron images), and the last term represents the direct impurity-electron interaction. For the case of the semiconductor/insulator/metal interface problem with a finite dielectric layer, where an infinite series of images arise, the above-proposed variational wave functions for the ground state as well as for the first excited states have been used. The assumption that the electron interacts only along the $z$ axis with all "infinite" number of images of the donor leads to the conclusion that the effect of all "infinite series" of the Coulomb interactions [the first and the second sum in Eq. (12)] can be described by one factor $f(z)=\exp (-\beta z)$ in the total trial wave function Eq. (3). We also use numerical finite difference approach to obtain numerically exact results for this problem.

In Fig. 8 we present the dependence of the ground state energy (in units of $2 R^{*}$ ) as a function of $d / a_{B}$, near a semiconductor/dielectric/metal interface with dielectric layer thickness $l / a_{B}=0.5$, using the trial function with three variational parameters (solid curve) and by stars is indicated the dependence found on the basis of the finite element techniques. The comparison between the results obtained with the variational function with three parameters and the exact results gives a relative error of $3 \%$ when $d=a_{B}$, thickness of dielectric layer $l=a_{B}$ (for the permittivity $\varepsilon_{1}=3.4$ of the ox- 


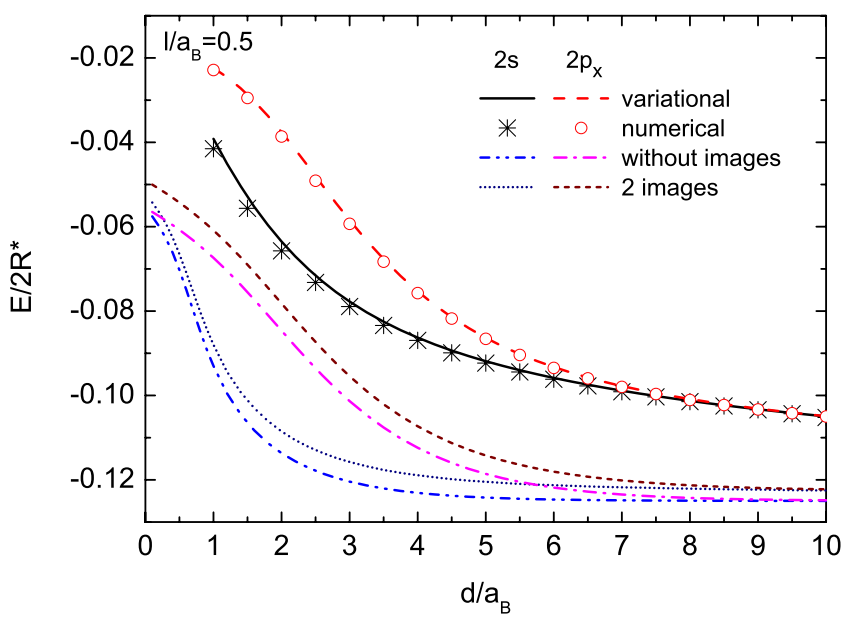

FIG. 9. (Color online) The dependence of the excited state energies $2 s$ (solid curve) and $2 p_{x}$ (dashed curve) calculated variationally and exactly indicated by stars for $2 s$ and by circles for $2 p_{x}$ state (in units of $2 R^{*}$ ) as a function of $d / a_{B}$ for a semiconductor/ dielectric/metal system with $l / a_{B}=0.5$. The excited state energies for $2 s$ (dot-dot-dashed curve) and $2 p_{x}$ (dot-dashed curve) for the system in the absence of the image effects, as well as $2 s$ (dotted curve) and $2 p_{x}$ (dashed curve) when for each of the donor and the electron we take only one image with respect to each of the metal and the dielectric interfaces.

ide layer and $\varepsilon_{3}=11.9$ for the semiconductor), and the lowering due to the factor $f(z)$ is about $7.6 \%$. For $d=a_{B}$, and dielectric layer thickness $l=a_{B} / 2$ the relative error becomes $3.8 \%$, and the lowering due to the factor $f(z)$ is about $8.5 \%$ now. We see that with the decrease in the dielectric layer thickness, when the influence of the metal gate becomes stronger the relative error increases and the influence of the image term $f(z)$ leads to a stronger energy lowering. In Fig. 8 we also present the ground state energy dependence (for $\left.l / a_{B}=0.5\right)$ for the case, when in the sum of Eq. (12) we retain only the $n=0,1$ terms, i.e., only one image charge for each real charge is included for each dielectric layer (see dot-dotdashed curve). Notice that this leads to an overestimation of the binding energy which for small $d$ values becomes almost a factor of 3 .

When $l>a_{B}$ the energies of the system with the finite dielectric layer tend to the results of the semiconductor/ dielectric interface case obtained with the potential Eq. (9). For opposite case of $l \ll a_{B}$ we obtain the results we found with potential Eq. (1).

Figure 9 shows the dependence of the excited state energy $(2,0,0)$ calculated variationally (solid curve) and "exactly" indicated by stars (in units of $2 R^{*}$ ) as a function of $d / a_{B}$ for the semiconductor/oxide/metal system with $l / a_{B}=0.5$, as well as for $(2,1,1)$ state calculated exactly (circles) and variationally (dashed curve). Notice that for $d>8 a_{B}$ the $2 s$ and $2 p_{x}$ states become degenerate. In Fig. 9 we compared the results also with the cases in which we neglected the image charge effect completely (dashed-dotted curves) and when we include the image charge approximately, namely, when only one image charge is included for each charge for each dielectric material (dashed and dotted curves). We found that for small $d$ values the binding energy is overestimated with

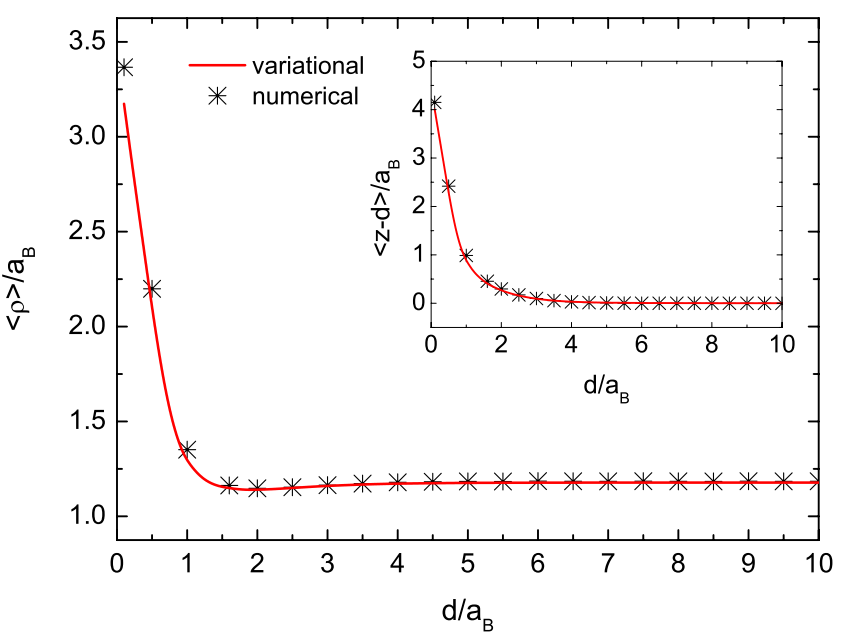

FIG. 10. (Color online) The average value of the electron position on the $\rho$ direction in the $1 s$ state vs the donor position in units of $a_{B}$ for the semiconductor/dielectric/metal interface. Inset: the dependence of the average distance between the electron and the donor along the $z$ direction.

almost a factor of 3 if the image charge effect is neglected [and if it is approximated by only the $n=0$ terms in Eq. (12)].

Figure 10 shows the average values of the electron position along the $z$ axis and the $\rho$ plane vs the donor position in units of $a_{B}$ for the semiconductor/dielectric/metal interface in the $1 s$ state. Notice that the qualitative behavior of both functions is similar to what was found in Fig. 5 with the quantitative difference that the minimum in $\langle\rho\rangle$ found for previous case is more pronounced.

Figure 11 shows the ground state energy dependence as a function of $d / a_{B}$ for different oxide layer thicknesses. For small values of the oxide layer thickness $l$ as a limiting case we obtain the energy of the semiconductor/metal system; for $l=0.1 a_{B}$ and $d=a_{B}$ we find that $E / 2 R^{*} \approx-0.15$, which is close to the ground state energy of the semiconductor/metal

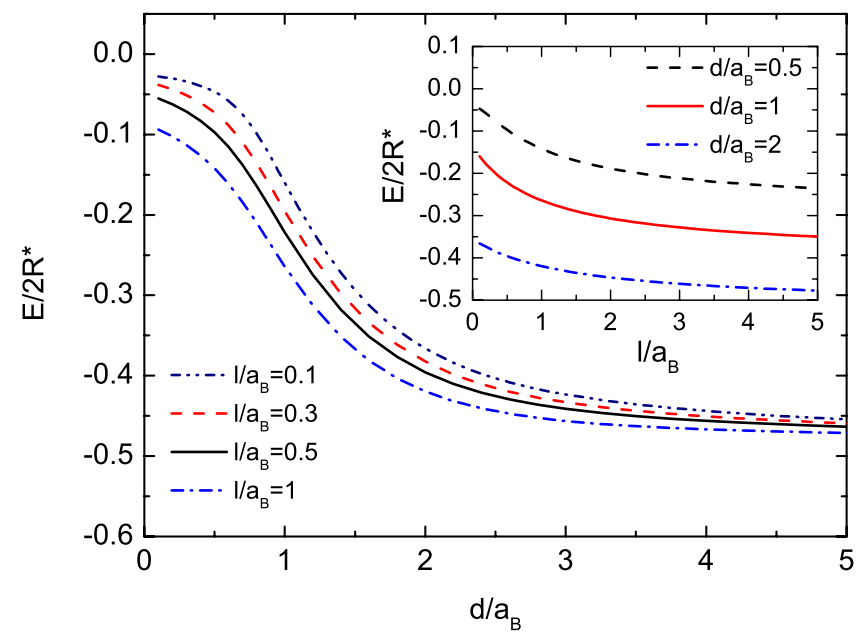

FIG. 11. (Color online) The ground state energy as a function of $d / a_{B}$ for a semiconductor/dielectric/metal system with different thicknesses of the oxide layer: $l / a_{B}=0.1,0.3,0.5$, and 1. Inset: the ground state energy as a function of $l / a_{B}$ for fixed $d / a_{B}=0.5,1$, and 2 . 


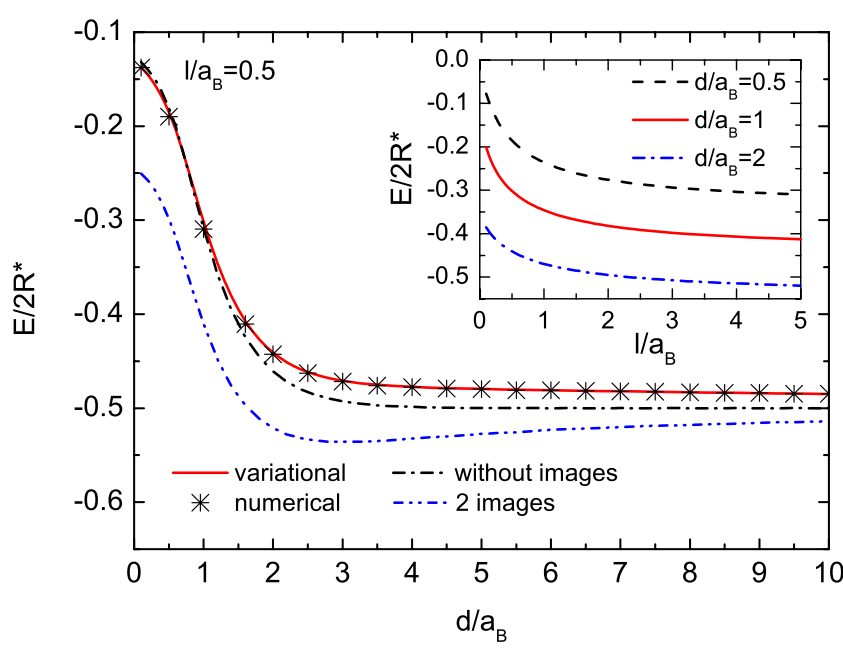

FIG. 12. (Color online) The dependence of the ground state energy (in units of $2 R^{*}$ ) as a function of $d / a_{B}$ with respect to the semiconductor/vacuum/metal interface $\left(\varepsilon_{o x}=1\right)$, with a vacuum layer thickness $l / a_{B}=0.5$, using the finite element techniques (stars) also from the trial function with three variational parameters (solid curve). The ground state energy in the absence of the image effects (dot-dashed curve), as well as for the case when for the donor and the electron we take only two images (dot-dot-dashed curve). Inset: the ground state energy as a function of $l / a_{B}$ for fixed $d / a_{B}=0.5,1$, and 2 .

system found for $d=a_{B}$ (see Fig. 1). In the opposite case when the oxide layer thickness is large, i.e., $l / a_{B} \geq 1$, we are close to the energies of the dielectric/semiconductor system presented in Fig. 4. The energy of the semiconductor/metal system at small $d$ is more shallow since the electron in this case is mainly bound with its image while in the semiconductor/dielectric case the electron feels the attraction of two positively charged motionless centers (donor and its image) which screen the repulsive interaction between the electron and its image resulting in a larger binding. In the same figure we present as an inset the dependencies of the ground state energy as a function of $l / a_{B}$ for fixed $d / a_{B}$ $=0.5,1$, and 2 . The energy of the system which contains only the direct electron-donor interaction, i.e., the image terms are absent, is $E_{D} / 2 R^{*}=-0.309$ for $d=a_{B}$. We find that the ground state energy of the semiconductor/dielectric/metal system for $d / a_{B}=1$ and $l \approx 1.5 a_{B}$ is equal to the energy of the system which contains only the direct electron-donor interaction. The image terms which arise due to the dielectric and the metallic interfaces compensate each other for these specific values of $d$ and $l$.

Finally, we investigated the ground as well as the first excited state energies of a semiconductor/vacuum/metal system with $\varepsilon_{o x}=1$. The obtained dependence for the ground state energy with a fixed vacuum layer thickness $l=0.5$ is shown in Fig. 12 with an inset figure of the ground state energy dependence on $l / a_{B}$ for fixed $d / a_{B}=0.5,1$, and 2 . Here we also find that the image terms due to the dielectric and the metallic interfaces compensate each other for values of $d=a_{B}$ and $l=0.5 a_{B}$ since the ground state energy (dotdashed) curve for the pure electron-donor problem crosses the energy of the system with an infinite number of images (solid curve) in Fig. 12.

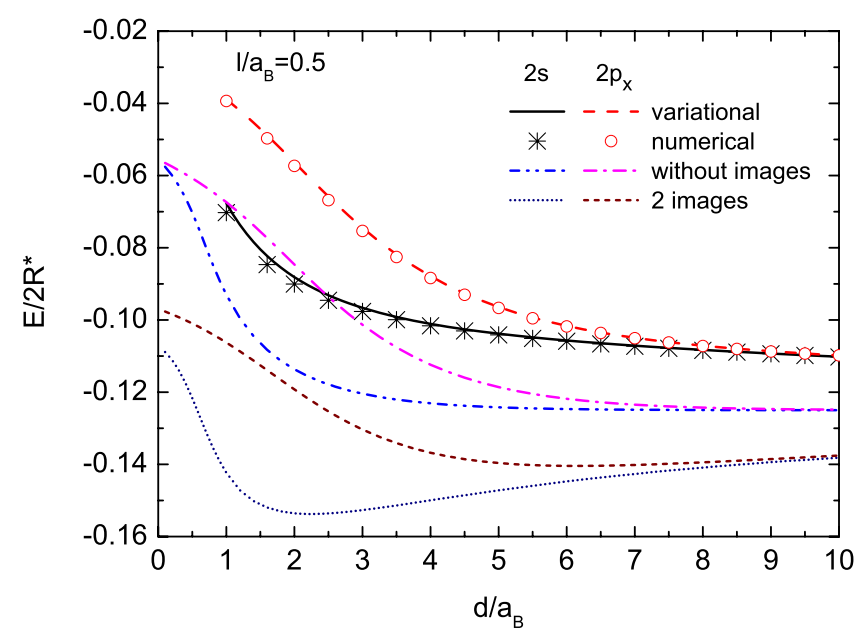

FIG. 13. (Color online) The dependence of the excited state energies $2 s$ (solid curve) and $2 p_{x}$ (dashed curve) calculated variationally and exactly indicated by stars for $2 s$ and by circles for $2 p_{x}$ state (in units of $2 R^{*}$ ) as a function $d / a_{B}$ for a semiconductor/ vacuum $/$ metal system $\left(\varepsilon_{o x}=1\right)$ with $l / a_{B}=0.5$. For the system in the absence of the image effects $2 s$ (dot-dot-dashed curve) and $2 p_{x}$ (dot-dashed curve), as well as $2 s$ (dotted curve) and $2 p_{x}$ (dashed curve) when for each of the donor and the electron we take only two images.

The results for the excited state energies as a function of $d=a_{B}$ for $l=0.5$ are presented in Fig. 13. Remarkably, the approximate result when we neglect the effect of image charges is closer to the numerical exact result as compared to the approximation in which we include only two image charges. This indicates that the effect of the images resulting from the donor and from the electron largely compensate each other for these specific values of $d$ and $l$. Such a metal/ vacuum/semiconductor interface problem is encountered when one uses a STM to investigate impurity states localized near a semiconductor surface as was done in, e.g., Ref. 17.

\section{CONCLUSION}

The proposed variational trial wave functions for an impurity electron near a semiconductor/metal interface give good agreement with an exact numerical treatment of the problem for the ground as well as the first excited states. For small distance between the donor and the metal interface $d$ $=a_{B}$ the factor $f(z)$ in the trial wave functions, which is responsible for the interaction of the electron with the images, leads to an energy lowering of about $15 \%$ and with a relative error for the ground state energy of about $6 \%$. For an impurity near a semiconductor/dielectric interface we obtain a better agreement with the exact results than for the metal interface case. The energy lowering due to the factor $f(z)$ is about $5 \%$ for the semiconductor/dielectric system.

For the case of the semiconductor/insulator/metal interface problem with a finite dielectric layer the aboveproposed variational wave functions for the ground state as well as for the first excited states have been used, and a good agreement with the exact results is found. We have found that in the three-layer system, where an infinite series of 
images arises, the presence of the metallic gate strongly affects the energetics of the system for an oxide layer thickness $l<a_{B}$. The energy lowering due to the factor $f(z)$ for small distances, i.e., $d \simeq a_{B}$ and for $l<a_{B}$ is considerable.

We found that the effect of the image charges is large for small $d$ values and can lead to a factor of 2-3 difference in the binding energy of the ground and excited states. Only for the semiconductor/insulator/metal interface at some specific values of $l$ and $d$ this correction is almost neglectable because of a cancellation of the effect of the different images. For large $d$ values the convergence of the results without images toward the numerical exact result is slow, i.e., the difference exhibits a $1 / d$ power-law dependence.

Our calculations, which were performed within the effective mass approximation, lead to a decrease in the donor binding energy with decreasing distance of the donor to the interface. This general trend is found for a metallic/ semiconductor interface, for a dielectric/semiconductor interface (although here a small increase in the binding energy is found for $d / a_{B}>1$ ), and also for a metallic/dielectric/ semiconductor interface. These results are opposite to recent experimental results, ${ }^{18}$ where using a STM tip, the binding energy of $\mathrm{Si}$ donors in GaAs was found to increase with almost a factor of 7 when the donor was brought close to the GaAs interface. This discrepancy must be attributed to the discrete atomic character of $\mathrm{Si}-\mathrm{GaAs}$ system which was not included in our treatment of the problem. The latter may lead to modifications to our effective mass approach because the electron effective mass and the dielectric function may be different close to the GaAs surface. Furthermore, the Si donor locally distorts the GaAs lattice, in particular, when it is close to the surface, leading to strain fields which will have an effect on the electronic band structure close to the GaAs surface.

\section{ACKNOWLEDGMENTS}

This work was supported by the Belgian Science Policy (IAP) and the Brazilian Science Foundation CNPq. One of us (A.A.A.) is supported by the Belgian Federal Science Policy Office (IAP).

\footnotetext{
*francois.peeters@ua.ac.be

${ }^{1}$ H. Sellier, G. P. Lansbergen, J. Caro, S. Rogge, N. Collaert, I. Ferain, M. Jurczak, and S. Biesemans, Phys. Rev. Lett. 97, 206805 (2006).

${ }^{2}$ B. E. Kane, Nature (London) 393, 133 (1998).

${ }^{3}$ T. Schenkel, A. Persaud, S. J. Park, J. Nilsson, J. Bokor, J. A. Liddle, R. Keller, D. H. Schneider, D. W. Cheng, and D. E. Humphries, J. Appl. Phys. 94, 7017 (2003).

${ }^{4}$ T. Shinada, S. Okamoto, T. Kobayashi, and I. Ohdomary, Nature (London) 437, 1128 (2005).

${ }^{5}$ D. Jamieson, C. Yang, T. Hopf, S. Hearne, C. Pakes, S. Prawer, M. Mitic, E. Gauja, S. Andresen, F. Hudson A. S. Dzurak, and R. G. Clark, Appl. Phys. Lett. 86, 202101 (2005).

${ }^{6}$ J. L. O'Brien, S. R. Schofield, M. Y. Simmons, R. G. Clark, A. S. Dzurak, N. J. Curson, B. E. Kane, N. S. McAlpine, M. E. Hawley, and G. W. Brown, Phys. Rev. B 64, 161401(R) (2001).

${ }^{7}$ S. R. Schofield, N. J. Curson, M. Y. Simmons, F. J. Ruess, T. Hallam, L. Oberbeck, and R. G. Clark, Phys. Rev. Lett. 91, 136104 (2003).

${ }^{8}$ A. F. Slachmuylders, B. Partoens, F. M. Peeters, and W. Magnus, Appl. Phys. Lett. 92, 083104 (2008).
}

${ }^{9}$ M. J. Calderón, B. Koiller, and S. Das Sarma, Phys. Rev. B 75, 125311 (2007).

${ }^{10}$ D. B. MacMillen and U. Landman, Phys. Rev. B 29, 4524 (1984).

${ }^{11}$ M. Kumagai and T. Takagahara, Phys. Rev. B 40, 12359 (1989).

${ }^{12}$ A. A. Avetisyan, A. P. Djotyan, and E. M. Kazaryan, Phys. Status Solidi C 0, 734 (2003).

${ }^{13}$ R. Loudon, Am. J. Phys. 27, 649 (1959).

${ }^{14}$ A. Latge, N. Porras-Montenegro, and Luiz E. Oliveira, Phys. Rev. B 45, 9420 (1992).

${ }^{15}$ J. M. Shi, F. M. Peeters, and J. T. Devreese, Phys. Rev. B 51, 7714 (1995).

${ }^{16}$ A. A. Avetisyan, K. Moulopoulos, and A. P. Djotyan, Philos. Mag. 86, 2493 (2006).

${ }^{17}$ K. Teichmann, M. Wenderoth, S. Loth, R. G. Ulbrich, J. K. Garleff, A. P. Wijnheijmer, and P. M. Koenraad, Phys. Rev. Lett. 101, 076103 (2008).

${ }^{18}$ A. P. Wijnheijmer, J. K. Garleff, K. Teichmann, M. Wenderoth, S. Loth, R. G. Ulbrich, P. A. Maksym, M. Roy, and P. M. Koenraad, Phys. Rev. Lett. 102, 166101 (2009). 